
560-564.

\title{
Densidades de plantio na produtividade e qualidade de frutos de melancia
}

\author{
Anamaria RP Ramos'; Rita de Cássia S Dias²; Carlos Alberto Aragão ${ }^{1}$ \\ ${ }^{1}$ UNEB-DTCS, Av. Edgard Chastinet s/n, São Geraldo, 48900-000 Juazeiro-BA; ${ }^{2}$ Embrapa Semi-Árido, C. Postal 23, $56300-970$ \\ Petrolina-PE; anamaria-ramos@oi.com.br
}

\section{RESUMO}

O experimento foi conduzido na Embrapa Semi-Árido em Petrolina-PE, de setembro a dezembro de 2007, para avaliar o desempenho agronômico e a qualidade dos frutos de melancia cultivadas sob diferentes densidades de plantio. Foram avaliados três espaçamentos combinados com seis cultivares de melancia, sendo duas diplóides de polpa amarela (BRS Soleil e BRS Kuarah), duas triplóides (Híbrido Triplóide CPATSA e Extasy Seedless) e duas cultivares comerciais, também diploídes e de fruto pequeno (Smile e Sugar Baby). O delineamento experimental utilizado foi em blocos ao acaso, com esquema fatorial em três repetições. Os tratamentos resultaram da combinação de três espaçamentos $(2,0 \times 0,3 \mathrm{~m} ; 2,0 \mathrm{x}$ $0,4 \mathrm{~m}$ e $2,0 \times 0,5 \mathrm{~m}$ ) e seis cultivares de melancia. Foram realizadas duas colheitas, para o Hibrido Triplóide CPATSA e Extasy Seedless, aos 68 DAP (dias após o plantio) e 82 DAP. O primeiro e o segundo "corte", respectivamente, para as outras cultivares aconteceram aos 63 DAP e 77 DAP. Avaliaram-se a massa fresca da parte aérea (g), massa média de frutos $(\mathrm{g})$, produção comercial e refugo (g; diâmetro do fruto $(\mathrm{cm})$, comprimento do fruto $(\mathrm{cm})$, espessura de casca $(\mathrm{cm})$, teor de sólidos solúveis (SS), acidez titulável (AT), relação SS/AT e firmeza da polpa. As análises de variância foram realizadas usandose o teste $\mathrm{F}$ e as médias comparadas através do teste de Tukey ao nível de $5 \%$ de probabilidade. De modo geral, os espaçamentos não afetaram a qualidade dos frutos, de modo que o aumento da densidade de plantas permitiu a obtenção de maior produtividade.

\author{
ABSTRACT \\ Yield and quality of watermelon fruits cultivated under \\ different planting densities
}

The experiment was carried out in Petrolina, Pernambuco State, Brazil, from September to December 2007, to evaluate the agronomic performance and fruit quality of watermelon cultivated under different planting densities. Three planting spaces and six cultivars were evaluated, where two cultivars were of yellow pulp (BRS Soleil and BRS Kuarah), two cultivars were triploid (Triploid Hybrid CPATSA and Extasy Seedless) and two cultivars were commercial, of small fruits (Smile and Sugar Baby). The experimental design was a randomized block with a factorial scheme with three replications. There were evaluated two harvest moments for the Triploid Hybrid CPATSA and Extasy Seedless: 68 DAS (days after sowing) and 82 DAS. The first and second harvests of the other cultivars were carried out at 63 and 77 DAS, respectively. We evaluated the fresh weight of the aerial part $(\mathrm{g})$, average fruit weight $(\mathrm{g})$, commercial and noncommercial fruits yield $(\mathrm{g})$, fruit diameter $(\mathrm{cm})$, fruit length $(\mathrm{cm})$, rind thickness $(\mathrm{cm})$, total solid content (SS), titratable acidity (TA), the ratio between SS/TA and flesh firmness. The variance analyses were done by the F test and the means were compared by the Tukey test at $5 \%$. Different spacing did not affect the fruit quality, so that higher density of plants proportioned higher yields.

Keywords: Citrullus lanatus, spacing, crop management.

Palavras-chave: Citrullus lanatus (Thunb.), espaçamento, manejo.

(Recebido para publicação em 29 de setembro de 2008; aceito em 30 de outubro de 2009) (Received in September 29, 2008; accepted in October 30, 2009)

\begin{abstract}
$\mathrm{A}$ melancia (Citrullus lanatus (Thunb.) Matsum. \& Nakai) é uma Cucurbitaceae de grande expressão econômica e social, possuindo propriedades nutricionais e terapêuticas que aumentam o interesse do consumidor pelo seu fruto (Dias et al., 2006).

No Brasil, a preferência do mercado consumidor leva em consideração o tamanho e formato do fruto, coloração da polpa, teor de sólidos solúveis, presença ou ausência de sementes, principalmente. Mais recentemente destaca-se o surgimento de novos tipos de melancias, as chamadas mini-melancias, principalmente devido à exigência do mercado por frutos de menor tamanho, sem sementes e de excelente qualidade. Observa-se que poucos genótipos predominam na maior parte das lavouras,
\end{abstract}

sendo que a maioria é de frutos grandes, com massa média acima de 6,0 kg (Dias et al., 2006).

O desenvolvimento vegetativo tem importância para os produtores pois, associado à prolificidade, determina a densidade de plantio a ser escolhida e repercute no tamanho dos frutos e na produtividade. Segundo Resende \& Costa (2003), na densidade de plantio, as pressões exercidas pela população de plantas afetam o seu desenvolvimento.

Na melancia, o maior espaçamento entre plantas resulta em menor produtividade comercial com consequente aumento da massa média dos frutos (Halsey, 1959). Segundo Brinen \& Locascio (1979), a produtividade de frutos comerciais decresceu, ao passo que a massa média de fruto aumentou com o incremento do espaçamento entre plantas de $0,6 \mathrm{~m}$ para $2,4 \mathrm{~m}$. Srinivas et al. (1991) concluíram que a produtividade de frutos de melancia aumentou de 33,6 tha-1 para 38,9 $\mathrm{t} \mathrm{ha}^{-1}$ em $1984 \mathrm{e}$ de $30,3 \mathrm{t} \mathrm{ha}^{-1}$ para $36,2 \mathrm{t} \mathrm{ha}^{-1}$ em 1985 , quando a população de plantas passou de 11.111 para 16.666 plantas ha-1, respectivamente. Segundo Ne Smith (1993), a produtividade total e comercial de frutos de melancia aumentou quando o espaçamento entre plantas decresceu de 2,2 $\mathrm{m}$ para $0,9 \mathrm{~m}$.

Avaliando diferentes espaçamentos de plantio na produção de melancia cultivar Crimson Sweet no município de Petrolina, Rezende \& Costa (2003) verificaram que o incremento dos espaçamentos tanto entre linhas como entre plantas produziu frutos de maior 
tamanho, tendo o espaçamento 3,00 m $\mathrm{x} 0,80 \mathrm{~m}$ apresentado a maior massa fresca do fruto $\left(8,83 \mathrm{~kg}\right.$ fruto $\left.^{-1}\right)$ e o maior número de frutos por planta $(1,35$ frutos). A maior produção foi encontrada no espaçamento de 3,00 $\mathrm{m}$ entre linhas apresentando 42,46 t ha-1. Nos espaçamentos de 0,60 e 0,80 m entre plantas obtiveram as maiores produções com 42,50 e 45,29 $\mathrm{t} \mathrm{ha}^{-1}$, respectivamente, não mostrando diferenças entre si. $\mathrm{O}$ menor espaçamento entre plantas proporcionou maior produção de refugo (frutos abaixo de $6 \mathrm{~kg}$ ), com 20,21 t ha-1, seguido pelos espaçamentos de $0,60 \mathrm{~m}$ $\left(12,86 \mathrm{tha}^{-1}\right)$ e $0,80 \mathrm{~m}\left(8,62 \mathrm{t} \mathrm{ha}^{-1}\right)$.

Segundo Singh \& Naik (1989), características de qualidade de frutos na cultura da melancia como sólidos solúveis não foram influenciadas significativamente por espaçamentos e doses de nitrogênio, testados em dois ciclos da cultura.

Analisando alguns genótipos de melancia sem sementes (W 913 e SQ) no município de Mossoró, Santos Filha et al. (2005) analisaram e constataram diferenças significativas entre os genótipos para as características $\mathrm{pH}$, sólidos solúveis, firmeza e acidez titulável e tais diferenças podem ser atribuídas a aspectos intrínsecos de cada genótipo.

O objetivo deste trabalho foi avaliar o efeito do adensamento de plantio no desempenho e qualidade de cultivares de melancias de frutos pequenos.

\section{MATERIAL E MÉTODOS}

O experimento foi conduzido de setembro a dezembro de 2007, na Embrapa Semi-Árido, município de Petrolina$\mathrm{PE}$, coordenadas geográficas $09^{\circ} 09^{\prime}$ de latitude sul e $40^{\circ} 22^{\prime}$ de longitude oeste e altitude de $365,5 \mathrm{~m}$.

O solo em que foi desenvolvido o ensaio é classificado como LATOSSOLO VERMELHO-AMARELO Eutrófico plíntico, e a análise química do mesmo revelou os seguintes resultados: $\mathrm{pH}$ (água) $=6,8 ; \mathrm{CE}=0,37 \mathrm{dS} \mathrm{m}{ }^{-1} ; \mathrm{P}=49$ mg.dm ${ }^{-3} ; \mathrm{K}=0,37$ cmolc. $\mathrm{dm}^{-3} ; \mathrm{Ca}=$ 2,2 cmolc. $\mathrm{dm}^{-3} ; \mathrm{Mg}=0,9$ cmolc. $\mathrm{dm}^{-3}$; $\mathrm{Na}=0,05$ cmolc. $\mathrm{dm}^{-3} ; \mathrm{Al}=0,05$ cmolc. $\mathrm{dm}^{-3} ; \mathrm{H}+\mathrm{Al}=1,15$ cmolc. $\mathrm{dm}^{-3} ; \mathrm{MO}=$ 9,52 g. $\mathrm{kg}^{-1}$. Durante o experimento, as temperaturas máximas e mínimas, foram respectivamente 36,5 e $19,8^{\circ} \mathrm{C}$, a umidade relativa média foi de $51 \%$ e a precipitação total do período de $42,25 \mathrm{~mm}$.

O delineamento experimental utilizado foi de blocos ao acaso, com esquema fatorial com três repetições. Os tratamentos resultaram da combinação de três espaçamentos $(2,0 \times 0,3 \mathrm{~m} ; 2,0$ $\mathrm{x} 0,4 \mathrm{~m}$ e $2,0 \mathrm{x} 0,5 \mathrm{~m}$ ) e seis cultivares de melancia: Hibrido Triploíde CPATSA (T1); BRS Soleil (T2); BRS Kuarah (T3); Extasy Seedless (T4); Sugar Baby (T5) e Smile (T6). O tamanho total foi de $5,0 \times 2,0 \mathrm{~m}$, avaliando-se as plantas da parcela útil de $3,5 \times 2,0 \mathrm{~m}$. O número de plantas variou conforme o espaçamento dentro das linhas em 7, 8 e 11 plantas/ parcela.

O preparo do solo constou de uma aração a $30 \mathrm{~cm}$ de profundidade, seguida de duas gradagens e abertura de sulcos, onde foi feita a adubação de plantio com $30 \mathrm{~kg} \mathrm{ha}^{-1}$ de $\mathrm{N}, 120 \mathrm{~kg} \mathrm{ha}^{-1}$ de $\mathrm{P}_{2} \mathrm{O}_{5}$ e $60 \mathrm{~kg} \mathrm{ha}^{-1}$ de $\mathrm{K}_{2} \mathrm{O}$, além de 15 $\mathrm{kg} \mathrm{ha}^{-1}$ de sulfato de zinco e $10 \mathrm{~kg} \mathrm{ha}^{-1}$ de sulfato de cobre. Em cobertura foi aplicado, via fertirrigação, $40 \mathrm{~kg}$ de MAP $\left(21,8 \%\right.$ de $\mathrm{P}_{2} \mathrm{O}_{5}$ e $11 \%$ de $\mathrm{N}$, nas três primeiras aplicações), $94 \mathrm{~kg} \mathrm{ha}^{-1} \mathrm{de}$ $\mathrm{N}$ (na formulação de nitrato de cálcio: $15 \%$ de $\mathrm{N}$ e $20 \%$ de $\mathrm{Ca}$ ), aplicado até 50 dias após o plantio (DAP) e $53 \mathrm{~kg} \mathrm{ha}^{-1} \mathrm{de}$ $\mathrm{K}_{2} \mathrm{O}$ (sulfato de potássio), até 60 DAP. A irrigação foi por gotejamento, com gotejadores espaçados de 0,3 m e o manejo feito com base na evapotranspiração do dia anterior e feito com base na estação meteorológica da Embrapa, situada no campo experimental.

As irrigações foram feitas com base na evaporação de água do tanque classe A e no coeficiente de cultura (Kc). As irrigações foram iniciadas um dia antes do transplantio e ocorreram até dois dias antes da colheita, três vezes por semana.

A semeadura foi realizada em casa de vegetação, em bandejas de isopor, com 128 células, utilizando-se substrato comercial para hortaliças Plantmax ${ }^{\circledR}$, irrigadas diariamente, de maneira a manter o substrato sempre úmido até serem transplantadas para o campo, aos 12 dias.

A floração foi avaliada diariamente a partir da antese das primeiras flores femininas. O comprimento de ramo foi obtido com auxílio de uma fita métrica na data de abertura da primeira flor.

Os frutos foram colhidos aos 63 DAP (dias após o plantio), e aos 77 das cultivares BRS Soleil, BRS Kuarah, Sugar Baby e Smile e para Hibrido Triplóide CPATSA e Extasy Seedless, a colheita aconteceu aos 68 DAP e aos 82 DAP.

O ponto de colheita foi determinado de acordo com o ciclo da cultura. Após a colheita os frutos foram levados para o laboratório da Embrapa Semi-Árido.

Foram avaliados a massa fresca da parte aérea $(\mathrm{g})$; massa média de frutos (g); produção comercial (frutos sem deformação e tamanho próprio das cultivares) e refugo (frutos defeituosos e muito pequenos) ( $\mathrm{g})$; diâmetro do fruto $(\mathrm{cm})$; comprimento do fruto $(\mathrm{cm})$ e espessura de casca $(\mathrm{cm})$.

O teor de sólidos solúveis (SS), acidez titulável (AT) e firmeza da polpa foram obtidos em 3 frutos por parcela. O conteúdo de SS foi determinado por refratometria no ponto central da polpa. A relação entre SS e AT (RATIO), foi avaliada utilizado o valor do SS obtido no homogeneizado de toda polpa.

A acidez titulável (AT) foi determinada utilizando-se duas subamostras de cada material analisado, através de titulometria, sendo os resultados expressos em \% de ácido málico.

A firmeza de polpa foi obtida com penetrômetro manual, tipo CAT 719-20, com ponteira de diâmetro de $8 \mathrm{~mm}$ de diâmetro, sendo realizadas duas leituras, centro e lateral e os resultados expressos em Newton (N).

A espessura da casca foi determinada com emprego de paquímetro na região mediana do fruto.

$\mathrm{O}$ diâmetro e o comprimento dos frutos foram avaliados com auxílio de régua milimetrada. A caracterização dos frutos foi baseada na lista de descritores mínimos para melancia, citado por Diez et al. (2005).

As análises de variância dos dados foram realizadas através do teste $\mathrm{F}$ e as médias comparadas ente si através do teste de Tukey ao nível de 5\% de probabilidade, com auxilio do pacote estatístico SISVAR/UFLA (Ferreira, 
2003).

\section{RESULTADOS E DISCUSSÃO}

Verifica-se que todas as seis cultivares de melancia apresentaram formato arredondado, com cor de polpa característica para cada genótipo, sendo vermelho intenso para o Híbrido Triplóide CPATSA, Extasy Seedless, Sugar Baby e Smile, enquanto que as cultivares Soleil e Kuarah apresentaram polpa amarela. O padrão para número de sementes variou de forma marcante entre as cultivares, tendo a cv. Extasy e o híbrido triplóide CPATSA, um número reduzido de sementes quando comparados às demais cultivares. A cv. Sugar Baby não apresentou listras, com uma cor verde escuro na casca, conforme as características discriminadas pela empresa que a desenvolveu.

Constatou-se que a cor do fundo da casca é semelhante para todas as cultivares avaliada, havendo, no entanto, variação de tonalidades do fundo, ou seja, variação tendendo de clara para o híbrido triplóide CPATSA a muito escura para a cv. Sugar Baby.

Não se verificou diferença estatística para o comprimento de ramo principal das plantas de melancia, no momento da abertura da primeira flor.

Houve variação na precocidade dentre os genótipos testados: A cv. BRS Kuarah foi a mais precoce, com o florescimento médio em 35 dias, enquanto que a cv. Extasy Seedless foi a mais tardia com 42 dias até o aparecimento da primeira flor feminina. Dentre as cultivares mais precoces e a mais tardia, houve uma diferença de 7 dias, o que representa uma diminuição do ciclo, com a antecipação da colheita. As cultivares mais precoces são mais desejadas pela vantagem do menor tempo de colheita e conseqüentemente retorno mais rápido do investimento empregado no cultivo (Ferreira et al., 2003).

O número de frutos por planta variou de 2,5 a 3,0 frutos do menor espaçamento $(2,0 \times 0,3)$, para o maior $(2,0 \mathrm{x}$ $0,5)$. No entanto, no teste de médias não se detectou diferenças estatísticas para essa característica. Entre as cultivares testadas Sugar Baby e Extasy Seedless apresentaram maior número de frutos por planta $(4,4$ e 3,4$)$, a cv. Smile apresentou-se intermediária $(3,2)$ e as cvs. Triplóide CPATSA, BRS Soleil e BRS Kuarah tiveram os menores números de frutos por planta $(2,0$ e 1,8).

A massa fresca média da parte aérea das plantas foi de $6,87 \mathrm{~kg}$ para os três espaçamentos trabalhados; a massa média de frutos de $3,8 \mathrm{~kg}$ e a produtividade média total foi de $73,1 \mathrm{t}$, para os espaçamentos utilizados.
Uma das características que mais varia quando se trabalha com adensamentos é a massa média de frutos. No entanto, não foi observada alteração para essa característica nas cultivares e nos espaçamentos estudados (Tabela 1). Ainda na Tabela 1, constata-se que houve uma variação da massa fresca da parte aérea de plantas por parcela, quando se analisou as diferentes cultivares. A cv. Triplóide CPATSA apresentou superioridade para essa característica ao final do ensaio, com aproximadamente o dobro da massa foliar fresca das plantas e uma coloração verde ainda intensa. Provavelmente a explicação para este comportamento seja pela resistência do genótipo a oídio, uma vez que as demais cultivares apresentavam ataque severo do fungo.

A massa média dos frutos foi maior no híbrido triplóide CPATSA $(6,33 \mathrm{~kg})$, por se tratar de um genótipo selecionado para frutos médios.

Em espaçamento de plantio de 3,0 $\mathrm{m} \times 0,8 \mathrm{~m}$, este híbrido tem apresentado em média frutos acima de $10 \mathrm{~kg}$ (Dias et al., 2006), revelando uma diminuição no tamanho em função do aumento da densidade de plantio. Isto está de acordo com Resende \& Costa (2003) que, trabalhando com a melancia Crimson Sweet (frutos graúdos), verificaram que o menor espaçamento entre plantas

Tabela 1. Dados médios de número de frutos; massa fresca parte aérea ( $\left.\mathrm{kg}_{\text {parcela }}{ }^{-1}\right)$; massa de frutos $(\mathrm{kg})$; produtividade total $\left(\mathrm{kg}\right.$ ha $\left.{ }^{-1}\right)$ e produtividade comercial $\left(\mathrm{kg} \mathrm{ha}^{-1}\right)$ de cultivares de melancia plantadas em diferentes espaçamentos (average number of fruits, fresh mass of the aerial part $\left(\mathrm{kg} \mathrm{parcel}^{-1}\right)$; fruit mass $(\mathrm{kg})$; total yield $\left(\mathrm{kg} \mathrm{ha}^{-1}\right)$ and commercial yield $\left(\mathrm{kg} \mathrm{ha}^{-1}\right)$ of watermelon cultivars planted in different spacings). Petrolina, Embrapa Semi-Árido, 2007.

\begin{tabular}{lccccc}
\hline \multirow{2}{*}{ Espaçamento $(\mathbf{m})$} & $\begin{array}{c}\text { Frutos/planta } \\
\left(\mathbf{n}^{\mathbf{0}}\right)\end{array}$ & $\begin{array}{c}\text { Massa fresca p. aérea } \\
\left.\mathbf{( k g ~ p a r c e l a}^{-1}\right)\end{array}$ & $\begin{array}{c}\text { Massa de frutos } \\
\mathbf{( k g )}\end{array}$ & \multicolumn{2}{c}{ Produtividade } \\
\cline { 5 - 6 } & $2,5^{*} \mathrm{a}$ & $6,56 \mathrm{a}$ & $3,87 \mathrm{a}$ & $76,87 \mathrm{a}$ & $70,66 \mathrm{a}$ \\
\hline$(2,0 \times 0,3)$ & $2,9 \mathrm{a}$ & $7,25 \mathrm{a}$ & $3,92 \mathrm{a}$ & $80,59 \mathrm{a}$ & $74,15 \mathrm{a}$ \\
$(2,0 \times 0,4)$ & $3,0 \mathrm{a}$ & $6,80 \mathrm{a}$ & $3,67 \mathrm{a}$ & $61,85 \mathrm{a}$ & $58,59 \mathrm{~b}$ \\
\hline$(2,0 \times 0,5)$ & & & & \\
\hline Cultivares de melancia & & $13,83 \mathrm{~A}$ & $6,33 \mathrm{~A}$ & $86,58 \mathrm{~A}$ & $82,50 \mathrm{~A}$ \\
\hline Hib. Trip. CPATSA & $1,8 \mathrm{C}$ & $6,84 \mathrm{~B}$ & $4,57 \mathrm{~B}$ & $72,49 \mathrm{AB}$ & $68,75 \mathrm{AB}$ \\
BRS Soleil & $1,8 \mathrm{C}$ & $5,89 \mathrm{~B}$ & $4,02 \mathrm{~B}$ & $73,08 \mathrm{AB}$ & $66,88 \mathrm{AB}$ \\
BRS Kuarah & $2,0 \mathrm{C}$ & $4,88 \mathrm{~B}$ & $2,56 \mathrm{C}$ & $59,96 \mathrm{~B}$ & $52,97 \mathrm{~B}$ \\
Extasy Seedless & $3,4 \mathrm{AB}$ & $5,50 \mathrm{~B}$ & $2,46 \mathrm{C}$ & $74,34 \mathrm{AB}$ & $68,63 \mathrm{AB}$ \\
Sugar Baby & $4,4 \mathrm{~A}$ & $4,28 \mathrm{~B}$ & $2,98 \mathrm{C}$ & $72,05 \mathrm{AB}$ & $67,08 \mathrm{AB}$ \\
Smile & $3,2 \mathrm{~B}$ & 44,02 & 16,85 & 18,10 & 20,37 \\
\hline CV $(\%)$ & 23,88 & &
\end{tabular}

*Médias seguidas de mesma letra nas colunas não diferem significativamente pelo teste de Tukey a 5\% de probabilidade. 
Tabela 2. Dados médios de diâmetro $(\mathrm{cm})$; comprimento $(\mathrm{cm})$ e espessura de casca $(\mathrm{cm})$ de cultivares de melancia plantadas em diferentes espaçamentos (average diameter $(\mathrm{cm})$, length $(\mathrm{cm})$ and thickness of bark $(\mathrm{cm})$ of watermelon cultivars planted in different spacing). Petrolina, Embrapa Semi-Árido, 2007.

\begin{tabular}{lccc}
\hline Espaçamentos (m) & $\begin{array}{c}\text { Diâmetro } \\
\text { do fruto }(\mathbf{c m})\end{array}$ & $\begin{array}{c}\text { Comprimento } \\
\text { do fruto }(\mathbf{c m})\end{array}$ & $\begin{array}{c}\text { Espessura } \\
\text { de casca }(\mathbf{c m})\end{array}$ \\
\hline$(2,0 \times 0,3)$ & $19,07 \mathrm{a}$ & $20,14 \mathrm{a}$ & $1,01 \mathrm{a}$ \\
$(2,0 \times 0,4)$ & $18,82 \mathrm{a}$ & $20,28 \mathrm{a}$ & $0,99 \mathrm{a}$ \\
$(2,0 \times 0,5)$ & $18,57 \mathrm{a}$ & $19,82 \mathrm{a}$ & $0,95 \mathrm{a}$ \\
\hline Cultivares de melancia & & & \\
\hline Hib. Trip. CPATSA & $23,15 \mathrm{~A}$ & $24,62 \mathrm{~A}$ & $1,43 \mathrm{D}$ \\
BRS Soleil & $20,10 \mathrm{~B}$ & $21,34 \mathrm{~B}$ & $0,89 \mathrm{~B}$ \\
BRS Kuarah & $19,18 \mathrm{~B}$ & $20,67 \mathrm{~B}$ & $0,94 \mathrm{~B}$ \\
Extasy Seedless & $16,66 \mathrm{C}$ & $17,40 \mathrm{C}$ & $1,18 \mathrm{C}$ \\
Sugar Baby & $16,41 \mathrm{C}$ & $17,66 \mathrm{C}$ & $0,88 \mathrm{~B}$ \\
Smile & $17,43 \mathrm{C}$ & $18,78 \mathrm{C}$ & $0,58 \mathrm{~A}$ \\
\hline CV $(\%)$ & 5,85 & 6,60 & 14,46 \\
\hline
\end{tabular}

*Médias seguidas de mesma letra nas colunas não diferem significativamente pelo teste de Tukey a $5 \%$ de probabilidade.

proporcionou maior produção de frutos com menos de $6 \mathrm{~kg}$.

As cultivares Extasy Seedless, Sugar Baby e Smile tiveram a massa média em torno de $2,5 \mathrm{~kg}$. Já as cultivares de polpa amarela, com frutos de $4 \mathrm{~kg}$, foram intermediárias. Esses materiais foram escolhidos pelas suas plantas de pequeno porte e frutos pequenos, visando atender, tanto mercado externo como mercado interno (Tabela 1).

Garcia \& Sousa (2002), trabalhando com melancia cv. Crimson Sweet observaram uma diminuição linear nas massas médias de frutos totais e comerciais, à medida que ocorreu aumento na população de plantas, porém em melão, Faria et al. (2000) constataram que diferentes espaçamentos não interferiram na massa média dos frutos.

A produtividade comercial teve efeitos significativos independentes entre os fatores estudados (Tabela 3). As cultivares submetidas aos espaçamentos com 2,0 x 0,4 m e 2,0 x 0,3 m, apresentaram maiores produções $(80,59$ $\mathrm{t} \mathrm{ha}^{-1}$ e $76,87 \mathrm{t} \mathrm{ha}^{-1}$, respectivamente) e não apresentaram diferenças significativas entre si. Ao passo que as plantas submetidas ao maior espaçamento $(2,0$ $\mathrm{x} 0,5 \mathrm{~m}$ ), apresentaram menor produtividade $\left(61,85\right.$ t ha $\left.^{-1}\right)$ e diferença estatística para os espaçamentos acima citados. Resende \& Costa em 2003, trabalhando com a cultura melão, constataram a menor produtividade $\left(52,97 \mathrm{tha}^{-1}\right)$; as demais cultivares tiveram um comportamento intermediário (Tabela 1).

Diâmetro e comprimento de fruto, assim como espessura de casca não sofreram influência do adensamento; as diferenças ocorreram em função dos genótipos (Tabela 2). Provavelmente, a adubação utilizada no presente trabalho, que foi ajustada para a maior densidade de planta, atendeu ao aumento da demanda de nutrientes mesmo na mais elevada densidade de plantio. O hibrido CPATSA apresentou o maior diâmetro e comprimento de fruto, as cultivares BRS Soleil e Kuarah tiveram valores intermediários e Extasy Seedless, Sugar Baby e Smile apresentaram os menores valores para essas características. Para espessura de casca, na cv. Smile observou-se o menor valor $(0,58 \mathrm{~cm})$, seguido pela Sugar Baby, Soleil e Kurah. As cv. Extasy Seedless e Híbrido Triplóide CPATSA apresentaram a maior espessura de casca com 1,18 e 1,43 cm, respectivamente, o que confere maior resistência pós-colheita.

Não houve diferença significativa para sólidos solúveis (SS) (Tabela 3). O híbrido triplóide CPATSA apresentou o maior índice de SS com 9,07 e a cultivar Extasy Seedless, o menor valor $(6,88)$. Observou-se não haver influência do espaçamento sobre sólidos solúveis em melancia, concordando com resultados

Tabela 3. Médias de componentes de qualidade de frutos de cultivares de melancia plantadas em diferentes espaçamentos (average of quality components of watermelon fruits planted in different densities). Petrolina, Embrapa Semi-Árido, 2007.

\begin{tabular}{lcccc}
\hline Espaçamentos (m) & $\begin{array}{c}\text { SS } \\
\left({ }^{\circ} \text { Brix }\right)\end{array}$ & $\begin{array}{c}\text { AT } \\
(\% \text { de ac. } \text { málico) }\end{array}$ & $\begin{array}{c}\text { Ratio } \\
(\mathbf{S S} / \mathbf{A T})\end{array}$ & $\begin{array}{c}\text { Firmeza } \\
(\mathbf{N})\end{array}$ \\
\hline$(2,0 \times 0,3)$ & $7,72 * \mathrm{a}$ & $0,37 \mathrm{a}$ & $21,39 \mathrm{a}$ & $8,39 \mathrm{a}$ \\
$(2,0 \times 0,4)$ & $7,85 \mathrm{a}$ & $0,38 \mathrm{a}$ & $20,63 \mathrm{a}$ & $8,48 \mathrm{a}$ \\
$(2,0 \times 0,5)$ & $7,80 \mathrm{a}$ & $0,39 \mathrm{a}$ & $20,07 \mathrm{a}$ & $9,64 \mathrm{a}$ \\
\hline Cultivares de melancia & & & & \\
\hline Hib. Trip. CPATSA & $9,07 \mathrm{~A}$ & $0,38 \mathrm{ABC}$ & $23,73 \mathrm{~A}$ & $4,63 \mathrm{~B}$ \\
BRS Soleil & $7,95 \mathrm{~B}$ & $0,39 \mathrm{ABC}$ & $20,91 \mathrm{AB}$ & $4,22 \mathrm{~B}$ \\
BRS Kuarah & $7,80 \mathrm{BC}$ & $0,41 \mathrm{AB}$ & $19,30 \mathrm{~B}$ & $4,08 \mathrm{~B}$ \\
Extasy Seedless & $6,88 \mathrm{C}$ & $0,33 \mathrm{C}$ & $20,76 \mathrm{AB}$ & $19,01 \mathrm{~A}$ \\
Sugar Baby & $7,19 \mathrm{BC}$ & $0,34 \mathrm{BC}$ & $21,02 \mathrm{AB}$ & $6,58 \mathrm{~B}$ \\
Smile & $7,86 \mathrm{BC}$ & $0,43 \mathrm{~A}$ & $18,47 \mathrm{~B}$ & $14,52 \mathrm{~A}$ \\
\hline CV $(\%)$ & 8,96 & 12,70 & 11,51 & 20,63 \\
\hline
\end{tabular}

*Médias seguidas das mesmas letras minúsculas e maiúsculas nas colunas não difere entre si pelo teste de Tukey a $5 \%$ de probabilidade. 
obtidos por Singh \& Naik (1989).

Não se observou efeito de interação entre espaçamentos e cultivares para a acidez titulável. A acidez dos frutos das seis cultivares não variou em função dos espaçamentos (Tabela 3).

A acidez devida a ácidos orgânicos é uma característica importante no que se refere à palatabilidade de muitos frutos. Com poucas exceções, diminui com a maturação, em decorrência do processo respiratório ou de sua conversão em açúcares (Kader, 1978; Pretty, 1982).

A relação sólidos solúveis/acidez titulável (RATIO) não sofreu alteração com relação aos espaçamentos utilizados (Tabela 3). As diferenças encontradas foram inerentes de cada cultivar. Essa relação é uma das melhores formas de avaliação do sabor, sendo mais representativa que a medição isolada de açúcares ou da acidez, proporcionando boa idéia do equilíbrio entre esses dois componentes (Chitarra \& Chitarra, 2005).

Para algumas culturas já foi determinada a relação que proporciona melhor sabor do fruto. Em melão, a relação ideal é superior a 25:1 com a acidez igual ou inferior a 0,5\% (Cruess, 1973).

Na melancia, Garcia (1998) obteve relações que variam de 26,7 a 30:1, valores esses muito superiores aos observados neste trabalho.

Não se observou diferenças de firmeza da polpa dos frutos entre os espaçamentos trabalhados. As cultivares Extasy Seedless e Smile apresentaram as maiores firmezas 19,01 N e 14,52 N, respectivamente (Tabela 3 ).

De modo geral, os espaçamentos utilizados não afetaram a qualidade dos frutos de melancia, de modo que o aumento da densidade de plantas permitiu a obtenção de maior produtividade comercial, mas não total.

\section{REFERÊNCIAS}

BRINEN GH; LOCASCIO SJ. 1979. Plant and row spacing, mulch, and fertilizer rate effects on watermelon production. Journal of the American Society for Horticultural Science 104:724-726.

CHITARRA MI; CHITARRA AB. 2005. Póscolheita de frutos e hortaliças: fisiologia e manuseio. 2. ed. rev. e ampl. Lavras: UFLA. $785 \mathrm{p}$.

CRUESS WV. 1973. Produtos industriais de frutos e hortaliças. São Paulo: Edgard Blücher. 446p.

DIAS RCS; SILVA CMJ; QUEIRÓZ MA; COSTA ND; SOUZA FF; SANTOS MH; PAIVA LB; BARBOSA GS; MEDEIROS KN. 2006. Desempenho agronômico de linhas de melancia com resistência ao oídio. In: CONGRESSO BRASILEIRO DE OLERICULTURA, 46., 2006, Goiânia. Horticultura Brasileira 24:1416-1418. Suplemento. (CD ROM)

DIEZ MJ; DOOIJEWEERT W; MAGGIONI L; LIPMAN E. 2005. Appendix III. Minimum descriptor lists for Cucurbita, cucumber, melon and watermelon. In: REPORT OF A WORKING GROUP ON CUCURBITS, 1 ., 2005 Bulgaria. Report... Bulgaria: IPGRI. p.21-32. Disponível em: www.ecpgr.cgiar.org/ Workgroups/Cucurbits/Cucurbits_Plovdiv_ revdraft.pdf. Acesso em: 1 ago. 2007.

FARIA CM; COSTA ND; PINTO JM. 2000. Níveis de nitrogênio por fertirrigação e densidade de plantio na cultura do melão em um Vertissolo. Pesquisa Agropecuária Brasileira 35: 491-495.

FERREIRA DF. 2003. Sisvar versão 4.2. DEX/ UFLA.

FERREIRA MAJF; QUEIRÓZ MA; BRAZ LT. 2003. Correlações genotípicas, fenotípicas e de ambiente entre dez caracteres de melancia e suas implicações para o melhoramento genético. Horticultura Brasileira 21: 438442.

GARCIALF. 1998. Influência do espaçamento e da adubação nitrogenada sobre a produtividade da melancia no baixo Paraíba Piauiense. Teresina: Embrapa Meio Norte. 5p. (Comunicado Técnico, 79)

GARCIA LF; SOUZA VAB. 2002. Influência do espaçamento e da adubação nitrogenada sobre a produção da melancia. Revista de la Facultad de Agronomia 28: 59-70.

GRANGEIRO LC; PEDROSA JF; BEZERRA NETOF; NEGREIROS MZ. 1999. Rendimento de híbridos de melão em diferentes densidades de plantio. Horticultura Brasileira 17: 200206.

HALSEY LH. 1959. Watermelon spacing and fertilization. Proceedings Fla. State Horticulturae Society 72: 131-135.

KADER AA. 1978. Quality factors: definition and evaluation for fresh horticultural crops. In: KADER AA. Postharvest Technology of Horticultural Crops. California: University of California. p.118-121.

NESMITH DS. 1993. Plant spacing influences watermelon yield and yield components. HortScience 28: 885-887.

PRETTY KM. 1982. O potássio e a qualidade da produção agrícola. In: YAMADA T; IGUE K; MUZILLI O; USHERWOOD NR. Potássio na agricultura brasileira. Piracicaba: POTAFOS. p.177-194.

RESENDE GM; COSTAND. 2003. Características produtivas da melancia em diferentes espaçamentos de plantio. Horticultura Brasileira 21:695-698.

SANTOSFILHAMEC; MORAISFA;ANDRADE MEL; ARAÚJO JMM; AROUCHA EMM. 2005. Caracterização pós-colheita de híbridos de melancia sem semente nas condições de Mossoró-RN. In: CONGRESSO BRASILEIRO DE OLERICULTURA, 15., 2005, Fortaleza. Resumos... Fortaleza, SBO. (CD-ROM).

SINGH RV; NAIK LB. 1989. Response of watermelon (Citrullus lanatus Thumbs. Monsf.) to plant density, nitrogen and phosphorus fertilization. Indian Journal of Horticulture 46: 80-83.

SRINIVAS K; HEDGE DM; HAVANAGI GV. 1991. Effect of nitrogen fertilization and plant population on plant water relations, canopy temperature, yield and water use efficiency of watermelon (Citrullus lanatus). Singapore Journal of Primary Industries 19: 8-15. 